\title{
Developments in the mechanisms of allergy in 2018 through the eyes of Clinical and Experimental Allergy, Part I
}

\author{
Graham Roberts $^{1,2,3}$ | C. Almqvist ${ }^{4,5}$ | R. Boyle ${ }^{6}$ J. Crane ${ }^{7}$ | S.P. Hogan ${ }^{8}$ | \\ B. Marsland ${ }^{9}$ | S. Saglani ${ }^{10}$ | J. A. Woodfolk ${ }^{11}$
}

${ }^{1}$ Clinical and Experimental Sciences and Human Development and Health, Faculty of Medicine, University of Southampton, Southampton, UK

${ }^{2}$ NIHR Southampton Biomedical Research Centre, University Hospital Southampton NHS Foundation Trust, Southampton, UK

${ }^{3}$ The David Hide Asthma and Allergy Research Centre, St Mary's Hospital, Isle of Wight, UK

${ }^{4}$ Department of Medical Epidemiology and Biostatistics, Karolinska Institutet, Stockholm, Sweden

${ }^{5}$ Pediatric Allergy and Pulmonology Unit at Astrid Lindgren Children's Hospital, Karolinska University Hospital, Stockholm, Sweden

${ }^{6}$ Department of Paediatrics, Imperial College London, London, UK

${ }^{7}$ Department of Medicine, University of Otago Wellington, Wellington, New Zealand

${ }^{8}$ Department of Pathology, Mary H Weiser Food Allergy Center, Michigan Medicine, University of Michigan, Ann Arbor, MI, USA

${ }^{9}$ Department of Immunology and Pathology, Monash University, Melbourne, Vic.,

Australia

${ }^{10}$ National Heart \& Lung Institute, Imperial College London, London, UK

${ }^{11}$ Division of Asthma, Allergy and Immunology, Department of Medicine, University of Virginia School of Medicine, Charlottesville, VA, USA

\section{Correspondence}

Graham Roberts, Paediatric Allergy and Respiratory Medicine (MP803), Clinical and Experimental Sciences and Human Development in Health, Faculty of Medicine \& University Hospital Southampton, University of Southampton, Southampton, UK.

Email: g.c.roberts@soton.ac.uk

\begin{abstract}
In the first of two linked articles, we describe the development in the mechanisms underlying allergy as described by Clinical \& Experimental Allergy and other journals in 2018. Experimental models of allergic disease, basic mechanisms and clinical mechanisms are all covered.
\end{abstract}




\section{1 | EXPERIMENTAL MODELS OF ALLERGIC DISEASE}

\subsection{Immune mechanisms of allergic diseases}

Mast cells are a major allergic cell playing critical roles in the initiation and exacerbation of numerous allergic inflammatory processes including allergic asthma, atopic dermatitis (AD) and allergic rhinitis (AR) ${ }^{1-3}$ Engagement and cross-linking of IgE and the FceRI receptor on the surface of mast cells lead to the release of mast cell-derived mediators including histamine which drive vascular endothelial permeabilization, chemokine production, and intensification of the CD4+ type-2-dependent inflammatory response enhancing migration of inflammatory cells including eosinophils, macrophages and neutrophils to the site of reaction. ${ }^{2,4}$ Recently, clinical studies have demonstrated elevated levels of the pro-type-2 cytokine thymic stromal lymphopoietin (TSLP) in AR. ${ }^{5}$ TSLP is known to play an important role in the exacerbation of allergic phenotypes through regulation of mast cell function. ${ }^{6,7}$ Indeed, TSLP-deficient mice are resistant to anaphylactic shock. ${ }^{8}$ Employing a murine model of $A R$ investigators show that sensitization and intranasal challenge of mice with the egg antigen ovalbumin (OVA) induces nasal symptoms. ${ }^{9}$ The authors show that administration of the mast cell degranulating agent compound 48/80 induced symptoms of an allergic reaction that was associated with increased scratching behaviour and associated serum histamine levels. Notably, intraperitoneal injection (IP) of TSLP significantly evoked a stronger pruritic response that was sensitive to $\mathrm{H} 1$ receptor in an NK-1 receptor antagonism suggesting that TSLP-induced scratching behaviour was mediated by histamine, substance $P(S P)$ and PGD2. The demonstration that TSLP could exacerbate mast cell-mediated allergic reaction leads investigators to examine the effect of TSLP on in vitro human mast cells. TSLP stimulation of human cord blood-derived mast cells and human mast cell line $\mathrm{HMC}-1$ evoked an increase in intracellular $\mathrm{Ca}^{2+}$ and mast cell degranulation that was associated with increased p56 ${ }^{\text {lck }}$ activity. P56 ${ }^{\text {lck }}$ is a tyrosine kinase $\mathrm{p} 56^{\mathrm{lck}}$ that has previously been shown to be associated with mast cell-mediated allergic reactions. ${ }^{10}$ Pharmacological and genetical knock-down of p56lck activity inhibited the TSLPmediated increase in intracellular $\mathrm{Ca}^{2+}$ ion and human and mouse mast cell degranulation indicating that $\mathrm{p} 56^{\mathrm{lck}}$ is required for TSLPevoked mast cell histamine secretion. Employing p56 ${ }^{\text {lck }}$-deficient mice, the authors demonstrated that indeed p56 ${ }^{\text {lck }}$ deficiency abrogated histamine-driven allergic responses and mast cell-mediated swelling. Compelling mechanistic analyses revealed a requirement for $\mathrm{p} 56^{\text {lck }}$ and STAT6 interaction TSLP-induced mast cell degranulation and development of the AR symptoms. Importantly, the authors were able to provide supporting clinical data, showing increased levels of c-kit-positive p56 $6^{\text {lck}}$-positive cells in the nasal mucosa tissues of AR patients compared with control individuals. Also, the increase in the frequency of these cells was associated with heightened TSLP levels. Further studies are necessary to elucidate the possible relationship between p56 $6^{\text {lck }}$ STAT6 in driving TSLP-induced mast cell degranulation and histamine release.
Recently, hypochlorous acid $(\mathrm{HOCl})$ has been shown to possess broad spectrum antimicrobial activity as well as anti-inflammatory and wound-healing properties which has led to the development of $\mathrm{HOCl}$ formulations for the treatment of inflammatory skin disorders including pruritus skin wounds and atopic dermatitis. ${ }^{11,12}$ Early clinical evaluations have revealed that treatment of patients with various skin disorders including $\mathrm{AD}$ with $\mathrm{HOCl}$ is clinically beneficial; however, the underlying molecular basis of these effects remains unclear. ${ }^{11,12}$ Fukuyama et al $^{13}$ employing a murine model of $A D$ investigated the anti-pruritic and anti-inflammatory properties of $\mathrm{HOCl}$. Consistent with previous observations, the authors demonstrated that topical treatment of a $\mathrm{HOCl}$ hydrogel on $\mathrm{AD}$-like lesions in $\mathrm{NC} / \mathrm{Nga}$ mice reduced the development of lesions and evidence of itch (scratching bouts). The efficacy of $\mathrm{HOCl}$ at suppressing the clinical symptoms was comparable to betamethasone propionate (WHO class 2) topical corticosteroid ointment. The authors confirmed that previously described anti-inflammatory properties of $\mathrm{HOCl}$ showing that pre-treatment reduced inflammatory cytokine production, evidence of mast cell activation and IgE at affected skin tissue. However, the strongest anti-inflammatory impact of $\mathrm{HOCl}$ was observed on suppression of DC-derived IL-12 production which has important implications given the known transition of the $A D$ inflammatory response to CD4+ Th1 phase during the chronic phase of $A D$. Given that $A D$ is a chronic allergic skin disease characterized by inflammatory lesions and a chronic itch component, the authors also investigated the ability of $\mathrm{HOCl}$ to alter sensory responses in dorsal root ganglia (DRG) cultures. Interestingly, the authors show that $\mathrm{HOCl}$ impaired several pruritogenic agent-induced activation of DRG neurons revealing that $\mathrm{HOCL}$ could directly reduce the sensory response and thus significantly suppress DRG neuron activation. These studies suggest that $\mathrm{HOCl}$ can directly reduce sensory responses and significantly reduce $A D$-associated itch and inflammation.

\section{2 | Immune mechanisms of allergic asthma}

Previous mechanistic-based studies have revealed a role for ubiquitin-modifying enzyme TNFAIP3 (TNFa-induced protein 3, also known as A20) in the modulation of CD4+ IL-17+ T cells and myeloid cell-driven pulmonary neutrophil inflammation. ${ }^{14}$ TNFAIP3 expression in DCs is required for house dust mite (HDM)-specific TH17 cell differentiation through expression of the Th17-instructing cytokines IL-1 $\beta$, IL- 6 and IL-2314. This led to the speculation that the CD4+ T cell-derived IL-17 subsequently acted downstream on the myeloid cell compartment to drive the neutrophilic inflammatory response. In a follow-up study, Vroman and colleagues employing IL-17RAdeficient mice and myeloid cell-specific TNFAIP3-deficient mice (Lys $\mathrm{M}^{\text {cre }}$ Tnaip ${ }^{\mathrm{fl} / \mathrm{fl} \text { ) }}$ examined the requirement of IL-17RA signalling in activation of the myeloid compartment and induction of the neutrophil inflammatory response and asthma phenotype. The authors demonstrated that myeloid cell induction of neutrophil and eosinophilic inflammation in HDM-challenged mice was unaltered in the 
absence of IL-17RA signalling. ${ }^{15}$ The authors demonstrated that loss of IL-17RA signalling did not impact the production of pro-inflammatory cytokines, IL-1 $\beta$ and IL-6, and neutrophil chemoattractants CXCL1, CXCL2 and CXCL12 which are likely sufficient to sustain the neutrophilic response in the absence of IL-17RA signalling. Interestingly, the authors found that the absence of IL-17 RA signalling had limited effect of most components of the HDM-mediated allergic airway inflammatory response including Th2 differentiation and eosinophilic inflammation. This would suggest that a number of the IL-17 family members including IL-17A a IL-17 A/F IL-17F and IL-25 are dispensable in the induction of HDM-mediated Th2 eosinophilic inflammation. These data would suggest the existence of two independent pathways, IL-17RA dependent and independent in driving the myeloid cell-neutrophil and eosinophilic inflammation in the asthma phenotype.

Dendritic cells are thought to play an obligatory role in both the initiation and maintenance of the allergic inflammatory response of the lung and induction of the asthma phenotype. ${ }^{16,17}$ To gain better insight into the specific DC subsets that regulate components of the inflammatory response, Liu and colleagues performed segmental allergen challenge (SAC) on allergic individuals to phenotypically characterize the monocytoid and DC cell subsets in blood and bronchoalveolar lavage (BAL). ${ }^{18}$ The authors demonstrated that SAC induced an inflammatory cell infiltrate characterized by lymphocytes, monocytoid macrophages, neutrophils and eosinophils. Cytometry analysis revealed that the monocytoid macrophages were predominantly CD14+ BDCA4+, and DC:CD4+ T cell co-cultures revealed that mixed mononuclear population of the lung possessed comparable APC function to BDCA4+ plasmacytoid DC (pDC) from the blood. Interestingly, the authors observed that SAC induced preferential recruitment of allergen-specific $T$ cells to the lung. The authors concluded SAC induced the recruitment of monocytoid cells (CD14+ BDCA4+) to the lung with increased APC function. The monocytoid cells with enhanced APC function include circulating pDCs and monocytes transitioning into myeloid DCs likely driven by DC differentiation cytokines including IL-4, GM-CSF and TGFa which are induced by SAC. ${ }^{19}$ These studies suggest that allergen exposure is likely to promote the rapid recruitment of DCs and monocytes that possess the predominant APC function and drive the CD4-positive proliferative and Th2 effector function in the onset of the clinical manifestations of disease.

Subcutaneous and sublingual allergen immunotherapy (SCIT and SLIT) are effective modalities for the suppression of allergen-induced allergic manifestations providing long-term allergy relief. ${ }^{20,21}$ Recent clinical studies have reported differences in the kinetics and magnitude of the immunological changes induced by SCIT and SLIT ${ }^{22-24}$; however, the molecular basis by which SCIT and SLIT induce immunoprotective effects is currently unknown. Hesse et $\mathrm{al}^{25}$ employing a murine model of grass pollen (GP)-induced asthma and GP-specific SCIT and SLIT treatment protocol to unveil the immunological mechanisms by which SCIT and SLIT therapy drive immune tolerance and suppress symptoms of the asthma phenotype. The authors demonstrate that GP-specific SLIT treatment suppressed the development of allergen-induced AHR in the absence of any impact on the allergic inflammatory response. In contrast, GP-SCIT altered the immunological compartment stimulating dose-dependent decrease in BAL and lung eosinophil levels and CD4+ Th2 cytokine production (IL-5 and IL-13), high levels of GP-specific IgG1 and increased CD4+ Th1 response any dramatic impact on AHR. The predominant differences in these modes of AIT are route of allergy administration, duration of treatment and allergen dosage. SCIT is dependent on direct injection of high concentration of allergen for a short duration; in contrast, SLIT involves sublingual administration of low concentration of allergen for a sustained period. The difference in the immunological impact of these two immunotherapy modalities may be related to the targeted immune cells. SLIT has previously been shown to target oral $C D 11 b+C D 11 c-A P C s$ which are thought to possess strong tolerogenic capacity. ${ }^{26}$ In contrast, SCIT treatment is thought to preferentially promote allergen presentation by mPDCA+ CD11b+ pDCs25. It is possible that these dosage differences and preferential targeting of different DC populations are sufficient to alter the immune modulatory effects and activation of tolerogenic responses. These observations are consistent with other models of allergic airway disease whereby SLIT treatment has been shown to be effective at suppressing airway eosinophilia and AHR in the absence of any alteration in antigen-specific immunoglobulin. ${ }^{27}$ Furthermore, it is clear from clinical trials that SCIT treatment induces a more profound effect on the immunological component, driving a faster and stronger protective response. ${ }^{28}$ Interestingly that slit treatment is effective at suppressing the eosinophilic inflammation in the absence of any effect on AHR which dissociates the respiratory inflammatory response with the airways functional outcomes.

\section{3 | Immune-targeted approaches for the suppression of the allergic inflammatory response and suppression of the asthma phenotype}

Clinical and experimental evidence indicates that chronic airway inflammatory disease is driven by a CD4+ Th2 and/or Th17 inflammatory response. ${ }^{29}$ Recent dietary intervention studies have revealed that dietary factors including polyunsaturated fatty acids (eg omega-3), dietary fibres, short-chain fatty acids, vitamin D and vitamin D3 can suppress CD4+ Th2 and Th17 inflammation and protect against the allergic disease phenotype. ${ }^{30-34}$ One such dietary factor Curcumin, a yellow pigment in Indian spice turmeric, is a nutraceutical that possesses anti-inflammatory and antioxidant properties. ${ }^{34}$ Administration of Curcumin capsules to human asthmatics improved mean forced $\mathrm{FEV}_{1}$ and airway obstruction. ${ }^{35}$ However, the mechanism by which Curcumin modulates the allergic inflammatory response is unclear. Employing a murine model of OVA-induced asthma, Chen et al revealed that tetrahydrocurcumin (THC), a major active metabolite of Curcumin, is able to suppress pulmonary eosinophilia the airways remodelling (mucus hypersecretion and collagen deposition). ${ }^{36}$ The authors show that THC significantly reduced the level of CD4+ Th2 and Th17 cells in the allergic lung and this was 
associated with decreased levels of the cytokines, IL-13 and IL-17. Mechanistically, the authors show that suppression of the allergic phenotype was associated with suppression of both Th2-cell differentiation pathways, ${ }^{37-40}$ IL-4Ra-JAK-1-STAT- 6 and the Jagged1/ Jagged2-Notch-1/notch-2 pathway in the lung. THC was more effective than Curcumin in alleviating the nasal symptoms and suppressing the asthma phenotype and allergic airway inflammatory response (CD4+ Th2/ Th17 cells) and the airway remodelling phenotype. The greater efficacy of THC versus Curcumin is likely due to the increased bioavailability and plasma and tissue THC compared to Curcumin, and it is anticipated that THC should have a comparable safety profile to Curcumin.

Given the important role for eosinophils in driving the clinical manifestations of the asthma phenotype, ${ }^{41,42}$ there has been significant focus on development of therapeutic agents to inhibit eosinophil migration. One such approach has been targeting the chemokine receptor pathway predominately used by eosinophils to migrate into the respiratory tissues, namely the Eotaxin family (CCL11, CCL24 and CCL26)-CCR3 pathway. Gauvreau and colleagues evaluated the effect of repeated dose of the small molecule antagonist with high specificity for binding to CCR3 (AXP1275) on allergen-induced latephase response and the development of eosinophilia in patients with mild allergic asthma. The authors demonstrated that 50mg AXP1275 administered daily for 14 days to subjects with mild-to-moderate asthma was safe and well tolerated and there is no effect on the type, severity or frequency of treatment-emergent adverse events. Twelve days of AXP1275 treatment significantly increased methacholine PC20 compared with placebo which was lost post-allergen challenge. The authors did not observe any significant effect on the allergen-induced late-phase response or eosinophil levels in blood or sputum. Notably, AXP1275 plasma concentrations were variable and low at the time of allergen challenge which may have contributed to the poor efficacy; however, these results provide support for performing additional studies assessing enhanced exposure through higher and longer duration of compound exposure. The improved methacholine PC20 observed following 12 days of treatment in the absence of any profound effect on airway or circulating eosinophil levels highlights the possibility CCR3-dependent effects independent of eosinophil-mediated AHR. Previous studies have reported CCR3 expression on human lung smooth muscle cells and that CCR3 antagonists inhibit CCR3-muscarinic acetylcholine (M3) receptor-induced smooth muscle contraction. ${ }^{43}$

Neutrophil infiltration in sputum is associated with increased severe asthma phenotype. ${ }^{44,45}$ The molecular mechanisms underpinning the neutrophil-driven inflammatory response and severe asthmatic manifestations and refractory phenotype are not yet clear. $^{46,47}$ One neutrophil-derived protein thought to play a role in exacerbation of the asthma phenotype is neutrophil elastase. A study by Ogawa and colleagues describes a new mechanism whereby neutrophils induced smooth muscle hyperplasia via neutrophil elastase-induced fibroblast growth factor 2 (FGF2)-dependent process. Employing a murine model of HDM-induced asthma, investigators show that HDM challenge induces a mixed allergic inflammatory phenotype associated with the presence of eosinophils and neutrophils, elevated levels of IL-17A and airway remodelling including bronchial smooth muscle hyperplasia. Neutralization of IL-17A ablated the magnitude of the airway inflammation, neutrophil elastase activity and airway remodelling including smooth muscle hyperplasia suggesting a IL-17A-neutrophil-driven process. Antibody array studies revealed increased levels of FGF2 in whole lung homogenates from HDM-challenged mice and that increased levels of FGF2 positively correlated with increase in smooth muscle hyperplasia. Treatment of mice with the anti-FGF2 mAb attenuated the HDMinduced increase in smooth muscle thickness. Employing BEAS-2B cells and primary cultural bronchial epithelial cells, the authors showed that neutrophil elastase induced airway epithelial FGF2 expression and this was associated with increased airway epithelial cell detachment. Given that neutrophil elastase promoted detachment of epithelial cells, the investigators examined expression of epithelial adhesion molecules, E-cadherin and $\beta$-catenin and revealed that the mixed neutrophil eosinophil and elevated FGF2 asthma phenotype in the HDM-challenged mice was associated with significantly decreased epithelial E-cadherin and elevated nuclear translocation of $\beta$-catenin. Notably, the altered epithelial adhesion pathway was attenuated by IL-17A-neutralizing antibody treatment suggesting that $\mathrm{E}$-cadherin and $\beta$-catenin signalling in the epithelium was involved in the IL-17-neutrophil elastase stimulation of FGF2 production. To test this concept, investigators challenged mice with the neutrophil elastase inhibitor sivelestat and showed that neutrophil elastase inhibition was associated with reduced FGF-2 production, altered airway epithelial E-cadherin and $\beta$-catenin translocation and reduced bronchial smooth muscle thickness. These studies elegantly identify a mechanism whereby neutrophil elastase through FGF2 can promote airway remodelling and smooth muscle hypertrophy. The authors speculate that neutrophil elastase activation of the airway epithelium leads to altered $\mathrm{E}$-cadherin and $\beta$-catenin translocation which seems to promote FGF-2 production and smooth muscle hyperplasia. Taken together, these studies suggest that neutrophil elastase-epithelial FGF-2 axis may be a critical mechanism driving smooth muscle hypertrophy in neutrophil-dominant asthma.

The underlying molecular mechanisms involved in the recruitment of neutrophils into the respiratory tract during an asthma exacerbation have also been in intensive area of research focus. The chemokine interleukin-8 (IL-8) is thought to be a critical chemokine in the regulation of neutrophil migration. ${ }^{48}$ Indeed, interleukin eight levels are elevated in asthmatic patients and small molecule antagonists and inhibitors of the IL-8 receptor, CXCR2, have been shown to be effective in reducing neutrophil levels in the sputum and improvement in clinical symptoms in severe asthmatics. ${ }^{48,49}$ Recent studies have revealed that a $28-\mathrm{kDa}$ protein fragment of the alpha-3 chain of collagen IV (Tumstatin) plays an important role in the control of neutrophil inflammation in the asthmatic lung. The levels of Tumstatin are markedly reduced in adult asthmatics, and studies in experimental mouse asthma model revealed that recombinant Tumstatin results in significant amelioration of the asthma phenotype. ${ }^{50}$ These protein fragments have recently been shown to act independently of 
the parent ECM molecule and are collectively known as matrikines. ${ }^{51}$ Nissen et al $^{52}$ employed a mouse model to examine the efficacy of novel active region within the matrikine Tumstatin (CP17; a 17 amino acid fragment Tumstatin 65-82) in inhibiting neutrophil infiltration in the asthma phenotype. The authors show that treatment of neutrophils with CP17 inhibited reactive oxygen species (ROS) production and IL-8-mediated neutrophil migration in vitro. In vivo studies revealed that pre-treatment of mice with $\mathrm{CP} 17$ inhibited ovalbumin (OVA)-induced asthma exacerbations including neutrophil inflammation and airway remodelling including mucus hypersecretion. The effect of CP17 appeared to specifically inhibit neutrophil accumulation into the lung interstitium, and lumen as other inflammatory cells was unaffected. The sustained eosinophilic infiltrate likely explains the lack of effective CP17 on airway function. Previous reports suggest that collagen IV isoform 3 fragments can interfere with neutrophil function via blockade of interaction with the $\alpha_{v} \beta_{3}$-integrin pathway, ${ }^{53,54}$ which leads to the speculation that $\alpha_{v} \beta_{3}$-integrin may be a crucial pathway in interstitial migration of neutrophils into the airways during asthma exacerbations.

\section{4 | Glycosaminoglycans}

Glycosaminoglycans (GAGs) are a family of highly sulphated, complex, polydisperse linear polysaccharides coupled to core proteins that play an important role in regulating leucocyte migration from the blood vessel to the site of inflammation. GAGs including heparin/heparan sulphate, chondroitin sulphate/dermatan sulphate, keratan sulphate and hyaluronan have affinity for chemokines which prevents chemokine diffusion and degradation and permits the establishment of the necessary chemokine haptotactic gradient to permit directed leucocyte migration. Mechanistic studies have revealed an important role for glycosaminoglycans (GAGs) in establishment and maintenance of the chemokine haptotactic gradient required to promote leucocyte recruitment and migration to the site of inflammation. ${ }^{55-57}$ Experimental studies have demonstrated that GAGchemokine interactions are critical for chemokine activity in vivo. ${ }^{58}$ Given the importance of GAGs in the regulation of chemokine-mediated leucocyte migration, this mechanism has become an attractive target for therapeutic intervention to treat various inflammatory disorders. This has led to the development of mutant chemokine variants that have diminished chemokine receptor binding capacity, however retain GAG binding properties, generating a competitive inhibitor that competes with active chemokines for GAG interaction to diminish the chemokine concentration gradient and inhibit leucocyte migration. ${ }^{59-61}$ Repeated administration of allergens such as dinitrofluorobenzene (DNFB) or trinitrochlorobenzene (TNFB) to the skin of mice induces an inflammatory cell recruitment including DCs, CD4+ Th1 cells and neutrophils eliciting a CHS response that mimics human contact dermatitis. ${ }^{62}$ Previous reports suggest an important role for neutrophils in both the sensitization and elicitation phase of CHS in mice. ${ }^{63,64}$ Recent studies have demonstrated that CXCL974103 the $\mathrm{COOH}$ terminal peptide of human CXCL9 binds with high affinity to GAGs and inhibits CXCL8- and monosodium urate crystal (MSU)-induced neutrophil migration. ${ }^{65}$ In a follow-up study, Vanheule and colleagues examined whether the chemokine-GAG antagonist CXCL974-103 inhibited inflammation and neutrophildependent DFNB-induced contact hypersensitivity. ${ }^{66}$ The authors demonstrated that simultaneous treatment of mice with CXCL974103 and DNFB inhibited clinical symptoms of CHS, ear swelling and that this was associated with a reduction in neutrophil infiltration and the level of the neutrophil-derived proteolytic enzyme metalloproteinases MMP9. To define whether the antagonistic activity of the CXCL974-103 on neutrophil chemoattraction was through inhibition of CXCL6-GAG interactions, the authors examined CXCL6heparin interactions. Indeed, CXCL974-103 reduced CXCL6-heparin binding by twofold. Consistent with this, using an in vitro endothelial neutrophil migration essay the authors demonstrated that indeed CXCL974-103 inhibited CXCL6-induced neutrophil chemoattraction and migration across the endothelial layer. The authors showed that the CXCL974-103 competed with CXCL1, CXCL6 and CCL3 interaction with GAG thereby inhibiting CXCL6-mediated transendothelial neutrophil migration. Given the efficacy of the CXCL974-103 peptide in multiple different models of inflammation including $\mathrm{CHS}$ and gout, this provides significant hope for further development of therapeutic peptides or derivatives that antagonize chemokine-GAG interactions and inhibit leucocyte migration in inflammation.

\section{2 | BASIC MECHANISMS UNDERPINNING ALLERGY}

\section{1 | Short-chain fatty acids as modulators of the immune response}

Microbial metabolites are potent modulators of immune responses. Short-chain fatty acids (SCFAs), such as butyrate, propionate and acetate, are well-characterized metabolites produced by the microbiota in the colon by fermentation of dietary fibre. They promote local immunoregulation in the gut, but can also enter the circulation and influence haematopoiesis and peripheral immune responses. Most research has pointed towards an impact of SCFAs on myeloid cells, T cells or intestinal epithelial cells and colonocytes. ${ }^{67}$ Imoto et $\mathrm{al}^{68}$ have extended the potential impact of SCFA to airway epithelial cells. Using immunohistochemistry, they first revealed that two of the receptors for SCFA, GPR41 and GPR43, were expressed on airway epithelial cells. They next showed that exposing these cells to SCFAs, in particular propionate, induced tissue plasminogen activator ( $t$-PA) expression that was dependent upon both GPR41 and GPR43. t-PA is an important enzyme involved in the conversion of plasminogen to plasmin, which degrades cross-linked fibrin. This observation could have particularly relevance in chronic rhinosinusitis, where excessive fibrin deposition is associated with nasal polyps and low expression of $\mathrm{t}$-PA. Topically administered propionate could thus have the potential to treat patients with chronic rhinosinusitis by reducing fibrin deposition and consequently growth of nasal polyps. 


\section{2 | Eosinophils as immunomodulatory cells}

Recent advances in research of eosinophil biology have broadened the role of these granulocytes beyond end-stage effectors of allergic responses, to immunomodulatory cells with unique context-dependent characteristics and functionality. ${ }^{69}$ Geslewitz and colleagues compared eosinophils from mice challenged intranasally with Alternaria alternata $(\mathrm{A} a)$ allergens to those of mice transgenic for IL-5, which harbour high levels of eosinophils even at steady state. ${ }^{70}$ Remarkably, exposure to Aa allergens over a week led to persistence of eosinophils in the airways for nearly a month. This sustained eosinophilia was IL-13-dependent in vivo; however, ex vivo IL-13 conferred no survival benefit to the cells, indicating that in vivo it acts upon another cell type to indirectly support eosinophil survival. In addition to enhanced survival, the $A a$-induced eosinophils were cytokine enriched as compared to eosinophils from IL-5 transgenic mice indicating their potency for immunomodulation and tissue damage was far greater. ${ }^{70}$ Cañas and colleagues reported a novel mechanism through which eosinophils could play a pathogenic role in asthma. ${ }^{71}$ Eosinophils were isolated from healthy or asthmatic individuals, and exosomes were purified. When cultured with epithelial cells, the eosinophil exosomes from asthmatic individuals induced greater epithelial cell apoptosis, impaired wound repair and increased muscle cell proliferation. Although the exact mediators of these disease-promoting pathways were not described, transport of inflammatory mediators within exosomes could be a major mechanism of eosinophil effector function. Eosinophils are typically linked with the magnitude of allergic responses; however, there are also reports that eosinophils can elicit protective antiviral activity. ${ }^{72} \mathrm{Ma}$ et al utilized a mouse model of rhinitis to investigate whether nasal eosinophils could provide a level of protection against subsequent influenza infection. The nasal eosinophils, induced by repeated inoculation with small volumes of $A a$ that restricted it to the upper respiratory tract, did not protect mice against viral challenge; in fact, these mice exhibited an exaggerated susceptibility to mortality. This severe reaction was not due to an inability of the mice to control the virus, but rather was linked with an exaggerated inflammatory response characterized by IL-6 and neutrophils. ${ }^{73}$ Taken together with the Geslewitz et al study, ${ }^{70}$ the function and implications of eosinophils clearly need to be considered within the context of the tissue and local inflammatory environment as a whole.

\subsection{Dendritic cells mediating gender differences in asthma}

There are clear gender differences in severity and prevalence of asthma $^{74}$; however, little is known concerning the immunological basis of this phenomenon. Masuda et al investigated the role of dendritic cells in a mouse model as an underlying cause of increased type 2 inflammation in females. The authors report that there was an increase in the migration of $\mathrm{CD} 11 b^{\text {high }}$ and $\mathrm{CD} 103^{+}$
DCs to the draining lymph nodes in female mice as compared to male mice. The $\mathrm{CD}_{103^{+}} \mathrm{DC}$ s from female mice, in particular, exhibited an increased activation state and a more potent ability to activate CD4+ T cells to produce IL-4, IL-5 and IL-13. The authors linked this type 2 enhancing capacity of the $\mathrm{CD} 103^{+} \mathrm{DCs}$ to oestrogen, as $17 \mathrm{~b}$-oestradiol enhanced the phenomenon in vitro, identifying at least one pathway through which type 2 responses could be enhanced in females. ${ }^{73}$

\section{3 | CLINICAL MECHANISMS}

\section{1 | Filaggrin gene as mediators of progression from eczema to asthma}

Although eczema has been implicated in the development of asthma, the mechanisms for the sequential progression of atopic diseases are complex and continue to be debated. ${ }^{75,76}$ Mutations in the filaggrin gene, FLG, which encodes a protein pivotal to skin barrier integrity, were a seminal discovery in the aetiology of atopic dermatitis (AD). ${ }^{77}$ Chan et al explored the time-dependent relationship between lossof-function (LOF) mutations in FLG and risk of asthma in childhood in relation to eczema and aeroallergen sensitization. ${ }^{78}$ By applying a path analysis strategy to the Isle of White birth cohort, it was determined that the risk of asthma in later childhood that was linked to LOF in FLG passed through eczema and allergic sensitization occurring in the first few years of life. By contrast, LOF in FLG was directly linked to rhinitis. This study solidified the notion of increased skin permeability as an important driver of sensitization to aeroallergens and subsequent type 2 inflammation in the lower airways, but also highlighted the distinct events involved in the development of asthma and rhinitis.

\section{2 | Triggers for asthma attacks}

Other studies examined factors that trigger acute asthma episodes in childhood. Both aeroallergens and respiratory viruses are known to exacerbate asthma in children. ${ }^{5}$ Shrestha and colleagues looked at the impact of different pollen types according to daily ambient concentrations, in relation to 2098 children and adolescents hospitalized for asthma in South-West Sydney, Australia. ${ }^{79}$ A notable finding of that study was the increased risk of asthma among boys for grass, although grass, weeds and unclassified pollen were triggers for all children, with those between 2 and 5 years of age being most susceptible. In other work, Guibas et al studied 1207 children hospitalized for flu-like illness in order to assess how infection with influenza virus (IFV) differs from rhinovirus (RV) in relation to acute asthma. Not unexpectedly, those children who tested positive for RV also had increased wheeze and diagnosis of acute asthma. ${ }^{80}$ By contrast, IFV positivity in children was negatively associated with wheeze, whereas fever was more prominent. Nonetheless, those with a history of asthma 
experienced "asthma-augmented influenza" characterized by more severe systemic symptoms. This dichotomy highlighted the distinct presentation of acute illness with RV and IFV in children with and without a history of asthma.

\section{3 | Airway narrowing in asthma}

Two studies examined airway narrowing in asthma from quite different perspectives. The first focused on respiratory impedance, which has broad applicability as a tool for asthma management since it relies only on tidal breathing. Karayama tested how respiratory impedance relates to airway narrowing by imaging the asthmatic airways using quantitative computed tomography. ${ }^{81}$ The authors found that while respiratory resistance was correlated with reduced airway calibre in adults with asthma, respiratory reactance was not. Notably, respiratory impedance provided a sensitive tool, given that altered airway structure in lower branching bronchi and impaired impedance were present in those asthmatics who had normal spirometry. The second study by $\mathrm{Yu}$ et al addressed the cellular basis of airway narrowing from an epigenetic perspective. This tested the effect of a selective inhibitor of histone demethylation (GSK-J4) in a mouse model of allergic asthma, as well as on human airway smooth muscle cells (ASMC) in vitro. ${ }^{82}$ The results implicated epigenetic regulatory mechanisms in promoting asthma through effects on ASMC. Specifically, treatment with GSK-J4, which inhibits demethylation of histone $\mathrm{H} 3$ lysine 27, attenuated airway remodelling in vivo. Additionally, it reduced the proliferation of AMSC and their accumulation of contractile proteins in response to cytokines.

\subsection{The role of the innate immune response in immunological diseases}

From a cellular immunology angle, the role of the innate immune response in immunological diseases continues to garner much attention. Tsurikisawa et al explored the relationships between the Th2-promoting cytokines, IL-33 and TSLP, type 2 innate lymphoid cells (ILC2) and eosinophilic granulomatosis with polyangiitis (EGPA, also known as Churg Strauss Syndrome). Increases in blood ILC2 counts and serum IL-33 levels were linked to disease activity in EGPA. ${ }^{83}$ Notably, the different profiles of eosinophils and IL-33 at disease onset and relapse indicated involvement of these components at different stages of disease. Another study that focused on aspects related to mast cell biology provided evidence that the JAK1/JAK2 inhibitor ruxolitinib inhibited mast cell degranulation, as well as production of pro-inflammatory cytokines. ${ }^{84}$ This work built on recent evidence of a role for the JAK2-STAT5 pathway in mast cell activation and the beneficial effects of ruxolitinib in a few cases of mastocytosis. Although the study tested ruxolitinib in vitro in human mast cell lines, the results bolstered the rationale for pursuing clinical trials in this area. Finally, Lara-Marquez et al sought to distinguish bradykinin-mediated angioedema from histamine-mediated angioedema using a novel biomarker assay. ${ }^{85}$ This assay, which measured plasma kallikrein activity using a fluorometric substrate, identified those patients with angioedema who may be amenable to bradykinin-targeted medication, based on a threshold dose of dextran sulphate used to activate kallikrein. A unifying theme of these cellular studies is their relevance to strategies for targeted therapies in diverse inflammatory disorders.

\section{4 | CONCLUSIONS}

The year 2018 provided further advances in the field of allergy. ${ }^{86} \mathrm{We}$ look forward to publishing more interesting observations in 2020.

\section{ACKNOWLEDGEMENTS}

We would like to acknowledge the assistance of Catherine Hyland in putting together this review.

\section{CONFLICT OF INTEREST}

The author declare no conflict of interest

\section{REFERENCES}

1. Galli SJ, Gordon JR, Wershil BK. Mast cell cytokines in allergy and inflammation. Agents Actions Suppl. 1993;43:209-220.

2. Galli SJ, Tsai M. IgE and mast cells in allergic disease. Nat Med. 2012;18:693-704.

3. Galli SJ, Grimbaldeston M, Tsai M. Immunomodulatory mast cells: negative, as well as positive, regulators of immunity. Nat Rev Immunol. 2008;8:478-486.

4. Chai R, Liu B, Qi F. The significance of the levels of IL-4, IL-31 and TLSP in patients with asthma and/or rhinitis. Immunotherapy. 2017;9:331-337.

5. Ziegler SF, Roan F, Bell BD, Stoklasek TA, Kitajima M, Han H. The biology of thymic stromal lymphopoietin (TSLP). Adv Pharmacol. 2013;66:129-155.

6. He R, Geha RS. Thymic stromal lymphopoietin. Ann N Y Acad Sci. 2010;1183:13-24.

7. Han NR, Oh HA, Nam SY, et al. TSLP induces mast cell development and aggravates allergic reactions through the activation of MDM2 and STAT6. J Investig Dermatol. 2014;134:2521-2530.

8. Nam SY, Kim HY, Han NR, et al. Src-type tyrosine kinase p56lck is critical for thymic stromal lymphopoietin-induced allergic rhinitis. Clin Exp Allergy. 2018;49(11):875-889.

9. Kim MH, Jeong HJ. Damnacanthal inhibits the NF-kappaB/RIP-2/ caspase-1 signal pathway by inhibiting p56lck tyrosine kinase. Immunopharmacol Immunotoxicol. 2014;36:355-363.

10. Maarouf M, Shi VY. Bleach for atopic dermatitis. Dermatitis. 2018;29:120-126

11. Del Rosso JQ, Bhatia N. Status report on topical hypochlorous acid: clinical relevance of specific formulations, potential modes of action, and study outcomes. J Clin Aesthet Dermatol. 2018;11:36-39.

12. Fukuyama T, Martel BC, Linder KE, Ehling S, Ganchingco JR, Bäumer W. Hypochlorous acid is antipruritic and anti-inflammatory in a mouse model of atopic dermatitis. Clin Exp Allergy. 2018;48:78-88.

13. Vroman H, Bergen IM, van Hulst JAC, et al. TNF-alpha-induced protein 3 levels in lung dendritic cells instruct $\mathrm{TH} 2$ or $\mathrm{TH} 17$ cell differentiation in eosinophilic or neutrophilic asthma. J Allergy Clin Immunol. 2018;141:1620-1633.e1612. 
14. Vroman H, Das T, Bergen IM, et al. House dust mite-driven neutrophilic airway inflammation in mice with TNFAIP3-deficient myeloid cells is IL-17-independent. Clin Exp Allergy. 2018;48:1705-1714.

15. van Helden MJ, Lambrecht BN. Dendritic cells in asthma. Curr Opin Immunol. 2013;25:745-754.

16. Hammad H, Lambrecht BN. Dendritic cells and epithelial cells: linking innate and adaptive immunity in asthma. Nat Rev Immunol. 2008;8:193-204.

17. Liu MC, Xiao HQ, Breslin LM, Bochner BS, Schroeder JT. Enhanced antigen presenting and $T$ cell functions during late-phase allergic responses in the lung. Clin Exp Allergy. 2018;48:334-342.

18. Liu MC, Proud D, Lichtenstein LM, et al. Effects of prednisone on the cellular responses and release of cytokines and mediators after segmental allergen challenge of asthmatic subjects. J Allergy Clin Immunol. 2001;108:29-38.

19. Jacobsen L, Niggemann B, Dreborg S, et al. Specific immunotherapy has long-term preventive effect of seasonal and perennial asthma: 10-year follow-up on the PAT study. Allergy. 2007;62:943-948.

20. Calderon MA, Casale TB, Nelson HS, Demoly P. An evidence-based analysis of house dust mite allergen immunotherapy: a call for more rigorous clinical studies. J Allergy Clin Immunol. 2013;132:1322-1336.

21. Nelson HS. Subcutaneous immunotherapy versus sublingual immunotherapy: which is more effective? J Allergy Clin Immunol. 2014;2:144-149; quiz 150-141.

22. Dretzke J, Meadows A, Novielli N, Huissoon A, Fry-Smith A, Meads C. Subcutaneous and sublingual immunotherapy for seasonal allergic rhinitis: a systematic review and indirect comparison. J Allergy Clin Immunol. 2013;131:1361-1366.

23. Durham SR, Penagos M. Sublingual or subcutaneous immunotherapy for allergic rhinitis? J Allergy Clin Immunol. 2016;137:339-349. e310.

24. Hesse L, Brouwer U, Petersen AH, et al. Subcutaneous immunotherapy suppresses Th2 inflammation and induces neutralizing antibodies, but sublingual immunotherapy suppresses airway hyperresponsiveness in grass pollen mouse models for allergic asthma. Clin Exp Allergy. 2018;48:1035-1049.

25. Mascarell L, Saint-Lu N, Moussu H, et al. Oral macrophage-like cells play a key role in tolerance induction following sublingual immunotherapy of asthmatic mice. Mucosal Immunol. 2011;4:638-647.

26. Tourdot S, Airouche S, Berjont N, et al. Evaluation of therapeutic sublingual vaccines in a murine model of chronic house dust mite allergic airway inflammation. Clin Exp Allergy. 2011;41:1784-1792.

27. Bohle B. Immune mechanisms of SCIT and SLIT: facing possible differences? Clin Exp Allergy. 2014;44:304-306.

28. Foster PS, Maltby S, Rosenberg HF, et al. Modeling TH 2 responses and airway inflammation to understand fundamental mechanisms regulating the pathogenesis of asthma. Immunol Rev. 2017;278:20-40.

29. de Matos OG, Amaral SS, Pereira da Silva PE, et al. Dietary supplementation with omega-3-PUFA-rich fish oil reduces signs of food allergy in ovalbumin-sensitized mice. Clin Dev Immunol. 2012;2012:236564.

30. Weise C, Hilt K, Milovanovic M, Ernst D, Rühl R, Worm M. Inhibition of IgE production by docosahexaenoic acid is mediated by direct interference with STAT6 and NFkappaB pathway in human B cells. $J$ Nutr Biochem. 2011;22:269-275.

31. Trompette A, Gollwitzer ES, Yadava K, et al. Gut microbiota metabolism of dietary fiber influences allergic airway disease and hematopoiesis. Nat Med. 2014;20:159-166.

32. Smith PM, Howitt MR, Panikov N, et al. The microbial metabolites, short-chain fatty acids, regulate colonic Treg cell homeostasis. Science 2013;341:569-573.
33. Kunnumakkara AB, Bordoloi D, Padmavathi G, et al. Curcumin, the golden nutraceutical: multitargeting for multiple chronic diseases. Br J Pharmacol. 2017;174:1325-1348.

34. Abidi A, Gupta S, Agarwal M, Bhalla HL, Saluja M. Evaluation of efficacy of curcumin as an add-on therapy in patients of bronchial asthma. J Clin Diagn Res. 2014;8:Hc19-Hc24.

35. Chen JW, Kong Z-L, Tsai M-L, et al. Tetrahydrocurcumin ameliorates free fatty acid-induced hepatic steatosis and improves insulin resistance in HepG2 cells. J Food Drug Anal. 2018;26:1075-1085.

36. Chapoval S, Dasgupta P, Dorsey NJ, Keegan AD. Regulation of the T helper cell type 2 (Th2)/T regulatory cell (Treg) balance by IL-4 and STAT6. J Leukoc Biol. 2010;87:1011-1018.

37. Chen $\mathrm{XH}$, Patel $\mathrm{BK}$, Wang $\mathrm{LM}$, et al. Jak1 expression is required for mediating interleukin-4-induced tyrosine phosphorylation of insulin receptor substrate and Stat6 signaling molecules. J Biol Chem. 1997;272:6556-6560.

38. Chatila TA. Interleukin-4 receptor signaling pathways in asthma pathogenesis. Trends Mol Med. 2004;10:493-499.

39. Berkooz M, Pioline B, Rozali M. Closed strings in Misner space: cosmological production of winding strings. J Cosmol Astropart Phys. 2004;2004. https://doi.org/10.1016/j.molmed.2004.08.004

40. Amsen D, Antov A, Flavell RA. The different faces of Notch in T-helper-cell differentiation. Nat Rev Immunol. 2009;9:116-124.

41. O'Byrne PM, Naji N, Gauvreau GM. Severe asthma: future treatments. Clin Exp Allergy. 2012;42:706-711.

42. O'Byrne PM, Inman MD, Parameswaran K. The trials and tribulations of IL-5, eosinophils, and allergic asthma. J Allergy Clin Immunol. 2001;108:503-508.

43. Bacon $\mathrm{K}$, Moss $\mathrm{E}$, Bingham $\mathrm{K}$, et al. LATE-BREAKING ABSTRACT: a novel role for CCR3 in promoting airways hyperreactivity; Role for CCR3-muscarinic M3 receptor heterodimers. Eur Respir J. 2015;46:OA288.

44. Moore WC, Hastie AT, Li X, et al. Sputum neutrophil counts are associated with more severe asthma phenotypes using cluster analysis. J Allergy Clin Immunol. 2014;133:1557-1563 e1555.

45. Gibson PG. Inflammatory phenotypes in adult asthma: clinical applications. Clin Respir J. 2009;3:198-206.

46. Fahy JV, Kim KW, Liu J, Boushey HA. Prominent neutrophilic inflammation in sputum from subjects with asthma exacerbation. $J$ Allergy Clin Immunol. 1995;95:843-852.

47. Reddel HK, Taylor DR, Bateman ED, et al. An official American Thoracic Society/European Respiratory Society statement: asthma control and exacerbations: standardizing endpoints for clinical asthma trials and clinical practice. Am J Respir Crit Care Med. 2009;180:59-99.

48. Murphy PM. Neutrophil receptors for interleukin-8 and related CXC chemokines. Semin Hematol. 1997;34:311-318.

49. Gao H, Ying S, Dai Y. Pathological roles of neutrophil-mediated inflammation in asthma and its potential for therapy as a target. $J$ Immunol Res. 2017;2017:3743048.

50. Burgess JK, Boustany S, Moir LM, et al. Reduction of tumstatin in asthmatic airways contributes to angiogenesis, inflammation, and hyperresponsiveness. Am J Respir Crit Care Med. 2010;181:106-115.

51. Gaggar A, Weathington N. Bioactive extracellular matrix fragments in lung health and disease. J Clin Invest. 2016;126:3176-3184.

52. Nissen G, Hollaender H, Tang FSM, et al. Tumstatin fragment selectively inhibits neutrophil infiltration in experimental asthma exacerbation. Clin Exp Allergy. 2018;48:1483-1493.

53. Monboisse JC, Garnotel R, Bellon G, et al. The alpha 3 chain of type IV collagen prevents activation of human polymorphonuclear leukocytes. J Biol Chem. 1994;269:25475-25482.

54. Shahan TA, Ziaie Z, Pasco S, et al. Identification of CD47/integrin-associated protein and alpha(v)beta3 as two receptors for the 
alpha3(IV) chain of type IV collagen on tumor cells. Cancer Res. 1999;59:4584-4590.

55. Handel TM, Johnson Z, Crown SE, Lau EK, Proudfoot AE. Regulation of protein function by glycosaminoglycans-as exemplified by chemokines. Ann Rev Biochem. 2005;74:385-410.

56. Monneau Y, Arenzana-Seisdedos F, Lortat-Jacob H. The sweet spot: how GAGs help chemokines guide migrating cells. J Leukoc Biol. 2016;99:935-953.

57. Rajarathnam K, Sepuru KM, Joseph PRB, Sawant KV, Brown AJ. Glycosaminoglycan interactions fine-tune chemokine-mediated neutrophil trafficking: structural insights and molecular mechanisms. J Histochem Cytochem. 2018;66:229-239.

58. Proudfoot AE, Handel TM, Johnson Z, et al. Glycosaminoglycan binding and oligomerization are essential for the in vivo activity of certain chemokines. Proc Natl Acad Sci USA. 2003;100:1885-1890.

59. Gschwandtner M, Piccinini AM, Gerlza T, Adage T, Kungl AJ. Interfering with the CCL2-glycosaminoglycan axis as a potential approach to modulate neuroinflammation. Neurosci Lett. 2016;626:164-173.

60. Gschwandtner M, Strutzmann E, Teixeira MM, et al. Glycosaminoglycans are important mediators of neutrophilic inflammation in vivo. Cytokine. 2017;91:65-73.

61. Gschwandtner M, Trinker MU, Hecher B, et al. Glycosaminoglycan silencing by engineered CXCL12 variants. FEBS Lett. 2015;589:2819-2824.

62. Christensen AD, Haase C. Immunological mechanisms of contact hypersensitivity in mice. APMIS. 2012;120:1-27.

63. Christensen AD, Skov S, Haase C. The role of neutrophils and G-CSF in DNFB-induced contact hypersensitivity in mice. Immun Inflamm Dis. 2014;2:21-34.

64. Weber FC, Németh T, Csepregi JZ, et al. Neutrophils are required for both the sensitization and elicitation phase of contact hypersensitivity. J Exp Med. 2015;212:15-22.

65. Vanheule V, Janssens R, Boff D, et al. The positively charged $\mathrm{COOH}$ terminal glycosaminoglycan-binding CXCL9(74-103) peptide inhibits CXCL8-induced neutrophil extravasation and monosodium urate crystal-induced gout in mice. J Biol Chem. 2015;290:21292-21304.

66. Vanheule V, Crijns $H$, Poosti F, et al. Anti-inflammatory effects of the GAG-binding CXCL9(74-103) peptide in dinitrofluorobenzene-induced contact hypersensitivity in mice. Clin Exp Allergy. 2018;48:1333-1344.

67. Dang AT, Marsland BJ. Microbes, metabolites, and the gut-lung axis. Mucosal Immunol. 2019;12:843-850.

68. Imoto Y, Kato A, Takabayashi T, et al. Short-chain fatty acids induce tissue plasminogen activator in airway epithelial cells via GPR41\&43. Clin Exp Allergy. 2018;48:544-554.

69. Jacobsen EA, Lee NA, Lee JJ. Re-defining the unique roles for eosinophils in allergic respiratory inflammation. Clin Exp Allergy. 2014;44:1119-1136.

70. Geslewitz WE, Percopo CM, Rosenberg HF. Eosinophil persistence in vivo and sustained viability ex vivo in response to respiratory challenge with fungal allergens. Clin Exp Allergy. 2018;48:29-38.

71. Cañas JA, Sastre B, Rodrigo-Muñoz JM, et al. Eosinophil-derived exosomes contribute to asthma remodelling by activating structural lung cells. Clin Exp Allergy. 2018;48:1173-1185.

72. Percopo CM, Dyer KD, Ochkur SI, et al. Activated mouse eosinophils protect against lethal respiratory virus infection. Blood. 2014;123(5):743-752.
73. Masuda C, Miyasaka T, Kawakami K, et al. Sex-based differences inCD $103^{+}$dendritic cells promote female-predominant Th2 cytokine production during allergic asthma. Clin Exp Allergy. 2018;48:379-393.

74. Kynyk JA, Mastronarde JG, McCallister JW. Asthma, the sex difference. Curr Opin Pulm Med. 2011;17(1):6-11.

75. Davidson WF, Leung DY, Beck LA, et al. Report from the National Institute of Allergy and Infectious Diseases workshop on "Atopic dermatitis and the atopic march: Mechanisms and interventions.". J Allergy Clin Immunol. 2019;143:894-913.

76. Paller AS, Spergel JM, Mina-Osorio P, Irvine AD. The atopic march and atopic multimorbidity: many trajectories, many pathways. J Allergy Clin Immunol. 2019;143:46-55.

77. Palmer CN, Irvine AD, Terron-Kwiatkowski A, et al. Common loss-of-function variants of the epidermal barrier protein filaggrin are a major predisposing factor for atopic dermatitis. Nat Genet. 2006;38:441-446.

78. Chan A, Terry W, Zhang H, et al. Filaggrin mutations increase allergic airway disease in childhood and adolescence through interactions with eczema and aeroallergen sensitization. Clin Exp Allergy. 2018;48:147-155.

79. Paul AGA, Muehling LM, Eccles JD, Woodfolk JA. T cells in severe childhood asthma. Clin Exp Allergy. 2019;49:564-581.

80. Shrestha SK, Katelaris C, Dharmage SC, et al. High ambient levels of grass, weed and other pollen are associated with asthma admissions in children and adolescents: a large 5-year case-crossover study. Clin Exp Allergy. 2018;48:1421-1428.

81. Guibas GV, Tsolia M, Christodoulou I, Stripeli F, Sakkou Z, Papadopoulos NG. Distinction between rhinovirus-induced acute asthma and asthma-augmented influenza infection. Clin Exp Allergy. 2018;48:536-543.

82. Karayama M, Inui N, Mori K, et al. Respiratory impedance is correlated with airway narrowing in asthma using three-dimensional computed tomography. Clin Exp Allergy. 2018;48:278-287.

83. Yu Q, Yu X, Zhao W, et al. Inhibition of H3K27me3 demethylases attenuates asthma by reversing the shift in airway smooth muscle phenotype. Clin Exp Allergy. 2018;48:1439-1452.

84. Tsurikisawa N, Oshikata C, Watanabe M, Tsuburai T, Kaneko $\mathrm{T}$, Saito $\mathrm{H}$. Innate immune response reflects disease activity in eosinophilic granulomatosis with polyangiitis. Clin Exp Allergy. 2018;48:1305-1316.

85. Hermans MAW, Schrijver B, van Holten-Neelen C, et al. The JAK1/ JAK2- inhibitor ruxolitinib inhibits mast cell degranulation and cytokine release. Clin Exp Allergy. 2018;48:1412-1420.

86. Roberts G, Almqvist C, Boyle R, et al. Developments in the mechanisms of allergy in 2018 through the eyes of Clinical and Experimental Allergy, Part II. Clin Exp Allergy. 2019;49(12):1606-1621.

How to cite this article: Roberts G, Almqvist C, Boyle R, et al. Developments in the mechanisms of allergy in 2018 through the eyes of Clinical and Experimental Allergy, Part I. Clin Exp Allergy. 2019;49:1541-1549. https://doi.org/10.1111/ cea.13532 